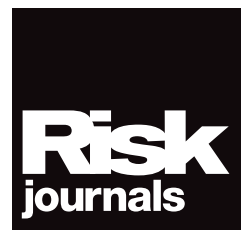

Journal of Computational Finance 21(1), 1-31

DOI: 10.21314/JCF.2016.325

Research Paper

\title{
Efficient estimation of sensitivities for counterparty credit risk with the finite difference Monte Carlo method
}

\author{
Cornelis S. L. de Graaf, ${ }^{1}$ Drona Kandhai, ${ }^{1,2}$ and \\ Peter M. A. Sloot ${ }^{1,3,4}$ \\ ${ }^{1}$ Computational Science, University of Amsterdam, Science Park 904, 1098 XH Amsterdam, \\ The Netherlands; emails: C.S.L.deGraaf@uva.nl, B.D.Kandhai@uva.nl, P.M.A.Sloot@uva.nl \\ ${ }^{2}$ Quantitative Analytics, ING Bank, PO Box 1800, 1000 BV Amsterdam, The Netherlands \\ ${ }^{3}$ National Research University ITMO, 49 Kronverksky Prospekt, St. Petersburg, 197101, Russia \\ ${ }^{4}$ Complexity Institute, S3 B2-A28 Nanyang Avenue, Singapore, 639798 Singapore
}

(Received December 16, 2014; revised July 14, 2015; accepted August 24, 2015)

\begin{abstract}
According to Basel III, financial institutions have to charge a credit valuation adjustment (CVA) to account for a possible counterparty default. Calculating this measure and its sensitivities is one of the biggest challenges in risk management. Here, we introduce an efficient method for the estimation of CVA and its sensitivities for a portfolio of financial derivatives. We use the finite difference Monte Carlo (FDMC) method to measure exposure profiles and consider the computationally challenging case of foreign exchange barrier options in the context of the Black-Scholes as well as the Heston stochastic volatility model, with and without stochastic domestic interest rate, for a wide range of parameters. In the case of a fixed domestic interest rate, our results show that FDMC is an accurate method compared with the semi-analytic COS method and, advantageously, can compute multiple options on one grid. In the more general case of a stochastic domestic interest rate, we show that we can accurately compute exposures of discontinuous one-touch options by using a linear interpolation
\end{abstract}


technique as well as sensitivities with respect to initial interest rate and variance. This paves the way for real portfolio level risk analysis.

Keywords: finite difference Monte Carlo (FDMC); credit valuation adjustment (CVA); barrier options; portfolio; exposure computation.

\section{INTRODUCTION}

Financial crises typically have various causes, but they often have one effect: the call to model more risk factors. Since 1987, we have known that the volatility used in option pricing is not constant and can be better modeled as a stochastic process itself (Heston 1993). More recently, since the Lehman collapse in 2008, measures have been taken to prevent loss of money from a worthless derivative due to counterparty default. Currently, having a single price for an option or financial derivative is therefore not sufficient; institutions also need to know the creditworthiness of their counterparty.

Regulators drafted the Basel III accords (Basel Committee on Banking Supervision 2010), which state that banks need to charge a premium to their trading counterparty for its creditworthiness. This is done via the so-called credit valuation adjustment (CVA), which adjusts the price of a derivative according to the creditworthiness of the counterparty. Moreover, additional capital requirements and limit-monitoring based on potential future losses should be in place. Computing these measures implies that the valuation and risk management of even straightforward plain vanilla options is a high-dimensional and complex problem.

In de Graaf et al (2014), we introduced the finite difference Monte Carlo (FDMC) method to calculate the exposure profiles of a derivative. This is done for Bermudan put options, which have an early exercise feature at preset discrete time points. Similar to the methods that appear in $\mathrm{Ng}$ and Peterson (2009) and $\mathrm{Ng}$ et al (2010), the FDMC method uses scenario generation from the Monte Carlo method. Option prices are computed on a grid, and the finite difference method and option values per path are obtained by interpolation on this grid. The expected exposure (EE) equals the mean of the resulting option price distribution, whereas the potential future exposure (PFE) is a quantile of this distribution. In practice, apart from EE and PFE, the sensitivities to market factors (such as spot value, interest rate and volatility) are required for hedging and controlling the counterparty credit risk (CCR) of derivatives portfolios.

In this paper, we extend our previous study. We again incorporate the highly relevant skew effect that is dominantly present in the foreign exchange (FX) market by choosing the Heston model to drive the underlying FX rate. In the case of constant interest rates, we consider the estimation of first- and second-order sensitivities with respect to the spot FX rate. In contrast with the widely used bump-and-revalue method, we propose a path-dependent estimator that is leveraging from the already estimated 
local sensitivities on the finite difference grid. A rigorous analysis is performed in the case of barrier options, which pose severe numerical challenges due to the knock-out feature that results in a discontinuous terminal condition. Similar discontinuities also arise in portfolios with instruments of different maturities, with the possibility of error propagation on the computation grid in time. Therefore, we analyze such portfolios specifically in this work. We validate our results by comparing them with the Monte Carlo-COS method (Shen et al 2013).

Next, we relax the assumption of a constant domestic interest rate by looking at the three-factor Heston Hull-White model. This implies the use of coarse grids for which the interpolation is vital. We therefore compare exposure quantities for discontinuous one-touch (OT) options computed by a linear or spline interpolation. Additionally, we discuss the applicability of the path-dependent sensitivities with respect to initial variance and domestic interest rate. Again, the bump-and-revalue method acts as a benchmark.

The outline of this paper is as follows. In Section 2, we describe CVA and its sensitivities with respect to the initial underlying values. Section 3 will be the core of this research, where we describe how to use the FDMC method, together with our adjustments, to measure the sensitivity of CVA, and how to extend it to handle multiple options. In Section 4, we present results for a number of test problems; in Section 5 , the conclusions are summarized.

\section{PROBLEM FORMULATION}

\subsection{CVA under the Heston Hull-White model}

In the Heston Hull-White model, the volatility and the domestic interest rate are modeled as a stochastic process, such that the volatility smile and interest rate dynamics can be captured. The three-dimensional dynamics are given by

$$
\begin{aligned}
\mathrm{d} S_{t} & =\left(R_{t}^{\mathrm{d}}-r^{\mathrm{f}}\right) S_{t} \mathrm{~d} t+\sqrt{V_{t}} S_{t} \mathrm{~d} W_{t}^{1}, \\
\mathrm{~d} V_{t} & =\kappa\left(\eta-V_{t}\right) \mathrm{d} t+\sigma \sqrt{V_{t}} \mathrm{~d} W_{t}^{2}, \\
\mathrm{~d} R_{t}^{\mathrm{d}} & =\lambda\left(\theta(t)-R_{t}^{\mathrm{d}}\right) \mathrm{d} t+\gamma \mathrm{d} W_{t}^{3}, \\
\mathrm{~d} W_{t}^{i} \mathrm{~d} W_{t}^{j} & =\rho_{i, j} \mathrm{~d} t \quad \text { for } i \neq j \in[1,2,3],
\end{aligned}
$$

where $r^{\mathrm{f}}$ is the foreign interest rate, $\kappa$ is the mean-reverting speed in the CoxIngersoll-Ross (CIR) process for the variance, $\eta$ is the level of the long-term mean and $\sigma$ is the so-called volatility of volatility. The domestic interest rate $R_{t}^{\mathrm{d}}$ follows

the Hull-White stochastic differential equation (SDE), where $\lambda$ is the mean-reverting speed, $\theta(t)$ is the level of the long-term mean deduced from the forward curve, and $\gamma$ is the volatility of the short rate. The SDEs are coupled by the correlated Wiener processes. Note that the same dynamics will hold for an equity derivative with stochastic 
interest rate and constant dividend. The FX rate is modeled in more detail if it is also assumed that the foreign interest rate is stochastic, which we do in a forthcoming research paper. The price $U$ of an option with maturity $T$ and payoff function $\phi\left(S_{T}, V_{T}, R_{T}^{\mathrm{d}}\right)$, and with the initial value of the underlying volatility and domestic short rate equal to $s, v$ and $r^{\mathrm{d}}$, respectively, equals

$$
U\left(s, v, r^{\mathrm{d}}, t_{0}\right)=\mathbb{E}\left[\mathrm{e}^{-\int_{t_{0}}^{T} R_{\xi}^{\mathrm{d}} \mathrm{d} \xi} \phi\left(S_{T}, V_{T}, R_{T}^{\mathrm{d}}\right) \mid S_{t_{0}}=s, V_{t_{0}}=v, R_{t_{0}}^{\mathrm{d}}=r^{\mathrm{d}}\right] .
$$

Because of the stochastic volatility and interest rate components, pricing formulas are three dimensional, and an analytic option price is harder to obtain, or not available. This is why numerical techniques such as the Monte Carlo or finite difference method are employed to solve the associated partial differential equation (PDE).

For risk purposes, it is obvious that one may be interested in the case where a loss is positive (a negative loss may be a profit); therefore, the exposure of an option at a future time $t<T$ is defined as

$$
\mathrm{E}(t):=\max \left(U\left(S_{t}, V_{t}, R_{t}^{\mathrm{d}}, t\right), 0\right),
$$

where $U\left(S_{t}, V_{t}, R_{t}^{\mathrm{d}}, t\right)$ is the value of a financial derivatives contract at time $t$.

The present $\mathrm{EE}$ at a future time $t<T$ is given by

$$
\mathrm{EE}(t):=\mathbb{E}\left[\mathrm{E}(t) \mid \mathcal{F}_{0}\right],
$$

where $\mathcal{F}_{0}$ is the filtration at time $t=0$. The discounted version of EE is computed as

$$
\mathrm{EE}^{*}(t):=\mathbb{E}\left[D(0, t) \mathrm{E}(t) \mid \mathcal{F}_{0}\right],
$$

where $D(0, t)$ is the discount factor. In this research, the expectation is calculated under the risk-neutral measure $\mathbb{Q} .{ }^{1}$ In the case of a long position in an option, the price (2.4) is always positive; thus, the EE (2.6) is equal to the future option price.

Another important risk assessment is given by the PFE. The quantiles $q=97.5 \%$ and $q=2.5 \%$ of the exposure distribution at time $t$ are defined as

$$
\operatorname{PFE}_{q}(t)=\inf \{x: \mathbb{P}(\mathrm{EE}(t) \leqslant x) \geqslant q\} .
$$

While computing CVA, we assume that the exposure and counterparty's default probability are independent. In the case of the discount factor, exposure and default probability all being independent, we can formulate the expression for CVA as follows (Gregory 2010):

$$
\operatorname{CVA}\left(t_{0}, T\right)=(1-\delta) \int_{t_{0}}^{T} \operatorname{EE}^{*}(t) \mathrm{d} \operatorname{PD}(t)
$$

\footnotetext{
${ }^{1}$ Typically, the future states can also be modeled under a real-world measure. This is possible when the FDMC method is used, but as this research focuses on the numerical applicability of this method, the risk-neutral measure $\mathbb{Q}$ is assumed.
} 
where $\delta$ is the recovery rate and $\operatorname{PD}(t)$ denotes the default probability of the counterparty at time $t$. The three essential elements are thus recovery rate, discounted EE and default probability.

In practice, CVA is hedged, and thus practitioners compute the sensitivity of the CVA with respect to its dependencies. We assume that the default probability is independent of exposure, such that the sensitivity with respect to $\Theta$ (where $\Theta$ can be $S_{0}, V_{0}$ or $R_{0}^{\mathrm{d}}$ ) can be rewritten as

$$
\begin{aligned}
\frac{\partial \operatorname{CVA}\left(t_{0}, T\right)}{\partial \Theta} & =\frac{\partial}{\partial \Theta}\left((1-\delta) \int_{t_{0}}^{T} \operatorname{EE}^{*}(t) \mathrm{d} \operatorname{PD}(t)\right) \\
& =(1-\delta) \int_{t_{0}}^{T} \frac{\partial \mathrm{EE}^{*}(t)}{\partial \Theta} \mathrm{d} \operatorname{PD}(t) .
\end{aligned}
$$

Following the same arguments, the second derivative with respect to $\Theta$ can be computed as

$$
\begin{aligned}
\frac{\partial^{2} \operatorname{CVA}\left(t_{0}, T\right)}{\partial \Theta^{2}} & =\frac{\partial}{\partial \Theta}\left((1-\delta) \int_{t_{0}}^{T} \frac{\partial \mathrm{EE}^{*}(t)}{\partial \Theta} \mathrm{d} \operatorname{PD}(t)\right) \\
& =(1-\delta) \int_{t_{0}}^{T} \frac{\partial^{2} \mathrm{EE}^{*}(t)}{\partial \Theta^{2}} \mathrm{dPD}(t) .
\end{aligned}
$$

By computing these sensitivities in this way, we need an efficient computation of the derivatives $\partial \mathrm{EE}^{*}(t) / \partial \Theta$ and $\partial^{2} \mathrm{EE}^{*}(t) / \partial \Theta^{2}$ for every $t \in\left[t_{0}, T\right]$.

To conclude, the CVA of a portfolio is determined by all the future mark-to-market (MtM) values of all the options in the portfolio (Basel Committee on Banking Supervision 2010). Further, if we want to compute the sensitivities, we also need the derivative at all future market scenarios. These requirements call for a valuation method that can compute option prices and derivatives for a wide range of market scenarios. In this paper, we will show that the FDMC method can compute these quantities quickly and accurately.

\section{COMPUTATION OF COUNTERPARTY EXPOSURE AND SENSITIVITIES}

\subsection{The FDMC method}

As presented in de Graaf et al (2014), the FDMC method uses the scenario generation of the Monte Carlo method and the pricing approach of the finite difference method. The market states are simulated by the quadratic exponential (QE) scheme (Andersen 2008). Next, a grid in the $s-, v$ - and $r^{\mathrm{d}}$-directions is created. This grid is chosen to be sufficiently large to capture all attained values of the scenario generation. On this grid, prices at any simulation date are calculated by the finite difference procedure. 
The specific state $\left(S_{m}, V_{m}, R_{m}^{\mathrm{d}}, t\right)$ is interpolated on the grid to obtain the option price $U\left(S_{m}, V_{m}, R_{m}^{\mathrm{d}}, t\right)$ at each path, for each time point. At every time point, the resulting future option values for all paths generate a distribution, and from this distribution the exposure profiles can be calculated. The EE can be obtained by averaging over all the prices at all the time points. The higher (97.5\%) and lower (2.5\%) PFEs can be computed by taking quantiles.

In the case of a path-dependent barrier option, if the underlying state hits the barrier level $B$, the option is exercised at this path, and the exposure for later time points is set to zero. The essential technique of modeling the exposure by the FDMC method can be presented as follows:

- generate scenarios/paths by Monte Carlo simulation;

- calculate option values and, for barrier options, check which paths hit the barrier;

- set the exposure at each path equal to the option value if the option is not exercised; otherwise, the exposure and all future exposures of this path are set equal to 0 ;

- compute the empirical distribution of the exposure at each exercise time;

- calculate EE, $\mathrm{PFE}_{2.5 \%}$ and $\mathrm{PFE}_{97.5 \%}$.

One important difference between the FDMC method and other approaches, such as the regression-based stochastic grid bundling method (SGBM) presented in Feng and Oosterlee (2014) or the Monte Carlo-COS method (Shen et al 2013), is that the FDMC method is directly applicable to non-affine models (eg, the stochastic alpha beta rho (SABR) or Heston Hull-White models with nonzero correlation between $S_{t}$ and $R_{t}^{\mathrm{d}}$ ). To compute exposures driven by non-affine models by the COS method or SGBM, an affine approximation is solved (see Feng and Oosterlee 2014; Guo et al 2013). This is not necessary when the FDMC method is used.

\subsection{The finite difference method}

The risk-neutral value $U$ at $t_{0} \leqslant T$ of a European option with maturity $T$ and payoff function $\phi$ can be expressed using the conditional expectation under the risk-neutral measure $\mathbb{Q}$, as follows:

$$
U\left(S_{t_{0}}, V_{t_{0}}, R_{t_{0}}^{\mathrm{d}}, t_{0}\right)=\mathbb{E}\left[\mathrm{e}^{-\int_{t_{0}}^{T} R_{u}^{\mathrm{d}} \mathrm{d} u} \phi\left(S_{T}\right)\right]
$$

where $\phi(\cdot)$ is the payoff function of the option. The finite difference procedure computes the price backward in time, starting at maturity $t=T$ and continuing to 
$t=t_{0}$. Thus, the pricing function $u$ is defined as a function of $\tau=T-t$, such that $u\left(S_{\tau}, V_{\tau}, R_{\tau}^{\mathrm{d}}, \tau\right)=U\left(S_{T-t}, V_{T-t}, R_{T-t}^{\mathrm{d}}, T-t\right)$. The Feynman-Kac theorem links the expectation (3.1) to the solution of a PDE by no-arbitrage arguments. This results in the following PDE:

$$
\frac{\partial u}{\partial \tau}=\boldsymbol{A} u,
$$

where, in the case of an FX option driven by the Heston Hull-White dynamics, the spatial differential operator $\boldsymbol{A}$ is given by

$$
\begin{aligned}
\boldsymbol{A} u=\frac{1}{2} & v s^{2} \frac{\partial^{2} u}{\partial s^{2}}+\frac{1}{2} \sigma^{2} v \frac{\partial^{2} u}{\partial v^{2}}+\frac{1}{2} \gamma^{2} \frac{\partial^{2} u}{\partial\left(r^{\mathrm{d}}\right)^{2}} \\
& +\left(r^{\mathrm{d}}-r^{f}\right) s \frac{\partial u}{\partial s}+(\kappa[\eta-v]) \frac{\partial u}{\partial v}+\left(\lambda\left[\theta(T-t)-r^{\mathrm{d}}\right]\right) \frac{\partial u}{\partial r^{\mathrm{d}}} \\
& +\rho_{1,2} \sigma v s \frac{\partial^{2} u}{\partial s \partial v}+\rho_{1,3} \gamma s \sqrt{v} \frac{\partial^{2} u}{\partial s \partial r^{\mathrm{d}}}+\rho_{2,3} \gamma \sigma \sqrt{v} \frac{\partial^{2} u}{\partial v \partial r^{\mathrm{d}}}-r^{\mathrm{d}} u
\end{aligned}
$$

Note that by setting $\gamma$ and $\lambda$ equal to 0 , we end up with the Heston PDE, where $r^{\mathrm{d}}$ is fixed. For a given state $\left(S_{\tau_{0}}, V_{\tau_{0}}, R_{\tau_{0}}^{\mathrm{d}}\right)$ at expiry, the payoff is known; in the finite difference method, this is used as an initial condition.

\section{Barrier options}

For a down-and-out barrier call or put option on an underlying $S_{\tau_{0}}$, with strike $K$ and barrier level $B$, the payoff function is equal to

$$
\phi\left(S_{\tau_{0}}\right)=\max \left(p\left(S_{\tau_{0}}-K\right), 0\right) \mathbf{1}_{\left\{S_{\tau_{0}}>B\right\}},
$$

with

$$
p= \begin{cases}1 & \text { for a call } \\ -1 & \text { for a put }\end{cases}
$$

The payoff function for European options can be obtained from this by setting $B=0$.

\section{One-touch options}

In the case of OT options, the holder receives a predetermined payout $H$ whenever the underlying reaches $K$, any time before maturity $T$. Thus, the payoff at expiry is given as

$$
\phi\left(S_{\tau_{0}}\right)=H \mathbf{1}_{\left\{S_{\tau_{0}} \geqslant K\right\}} .
$$




\subsubsection{Space discretization}

In the finite difference method, this PDE is solved on a finite set of points by discretizing in the $s-, v$ - and $r^{\mathrm{d}}$-directions. The domain to be discretized is chosen as $\left[0, S_{\max }\right] \times\left[0, V_{\max }\right] \times\left[-R_{\max }, R_{\max }\right]$, where $S_{\max }, V_{\max }$ and $R_{\max }$ are chosen to be sufficiently large to minimize the effect of the imposed boundary conditions, and such that all simulated market scenarios can be interpolated on the grid.

Let $s_{0}<s_{1}<\cdots<s_{m_{1}}, v_{0}<v_{1}<\cdots<v_{m_{2}}$ and $r_{0}<r_{1}<\cdots<r_{m_{3}}$ be the discretization in the $s$-, $v$ - and $r^{\mathrm{d}}$-directions, respectively, similarly to in Haentjens and In't Hout (2012). In all dimensions, the grid is chosen to be nonuniform. The $s$ dimension consists of a predefined interval $\left[S_{\text {left }}, S_{\text {right }}\right]$, in which points are uniformly spaced. $S_{\text {left }}$ and $S_{\text {right }}$ are chosen to contain the region of interest, ie, the region around the expected mean of the underlying. Following Haentjens and In't Hout (2012), for options without barriers we choose

$$
\left[S_{\text {left }}, S_{\text {right }}\right]=[0.5 K, K]
$$

Outside $\left[S_{\text {left }}, S_{\text {right }}\right]$, the points are distributed with the help of a hyperbolic sine function. In the barrier case, the nonuniform grid is chosen such that the dense region contains more than $95 \%$ of the non-exercised paths; generally, choosing

$$
\left[S_{\text {left }}, S_{\text {right }}\right]= \begin{cases}{[0.5 K, B]} & \text { for a up-and-out call or put, } \\ {[B, 1.5 K]} & \text { for a down-and-out call or put }\end{cases}
$$

is found to be sufficient. For a portfolio of options, however, we define $S_{\text {left }}$ and $S_{\text {right }}$ such that all possible strikes and barriers are included. In Figure 1, the two different nonuniform grids are shown.

In the $v$-direction, the grid is chosen similarly to in In't Hout and Foulon (2010). The grid is dense around $v=0$. We do this because, for realistic test parameters, the expected mean of the variance process is close to 0 . In addition, because the Heston PDE in the $v$-direction is convection-dominated close to 0 and the initial condition is non-smooth, numerical stability requires a high density of points in this region (Haentjens and In't Hout 2012).

Also, for the $r^{\mathrm{d}}$-direction, we follow Haentjens and In't Hout (2012). The grid is dense around 0 and stretched symmetrically toward the boundaries $-R_{\max }$ and $R_{\max }$ by using a sinus hyperbolic function.

The derivatives are approximated using central, forward and backward three-point stencils. All stencils are second-order accurate. For more details, we refer to Haentjens and In't Hout (2012). 
FIGURE 1 Nonuniform grids in the $s$-direction.

(a)

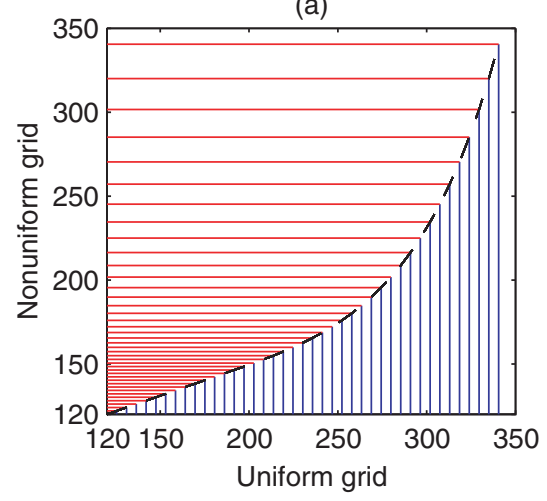

(b)

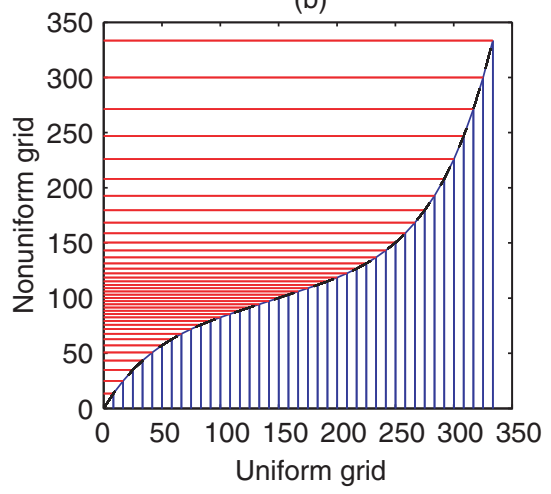

(a) A down-and-out put (DOP) option (where we choose $S_{\text {left }}=B=120$ and $S_{\text {right }}=140$ ). (b) A portfolio of options (with discontinuous points within $S_{\text {left }}=100$ and $S_{\text {right }}=150$ ).

TABLE 1 Boundary conditions and payoff functions under the Black-Scholes dynamics.

\begin{tabular}{lcc}
\multicolumn{1}{c}{ Option type } & $s \rightarrow S_{\max }$ & $s \rightarrow S_{\min }$ \\
\hline European call & $\frac{\partial u}{\partial s}=1$ & $u=0$ \\
European put & $u=0$ & $u=\mathrm{e}^{-\int_{\tau_{0}}^{\tau} r_{u}^{\mathrm{d}} \mathrm{d} u} K_{P}$ \\
Up-and-out barrier call & $u=0$ & $u=0$ \\
Up-and-out barrier put & $u=0$ & $u=\mathrm{e}^{-\int_{\tau_{0}}^{\tau} r_{u}^{\mathrm{d}} \mathrm{d} u} K_{P}$ \\
Down-and-out barrier call & $\frac{\partial u}{\partial s}=1$ & $u=0$ \\
Down-and-out barrier put & $u=0$ & $u=0$ \\
One-touch & $u=H$ & $u=0$ \\
\hline
\end{tabular}

\subsubsection{Boundary conditions}

The options considered in this research are of the following type: European call and put options with strike $K_{\mathrm{C}}$ and $K_{\mathrm{P}}$, respectively; barrier options with strike $K_{\mathrm{B}}$ and barrier level $B$, which can be down-and-out or up-and-out calls or puts; and OT options with strike $K_{\mathrm{O}}$ and payout $H$. The boundary conditions for the $s$ dimension used in this research are stated in Table 1 . Note that in the case of non-barrier options, $S_{\min }$ and $S_{\max }$ converge to 0 or $\infty$, respectively.

The boundary conditions in the volatility direction are imposed independently of the option type. In Ekström and Tysk (2011), it is shown that for a CIR process, 
such as the variance process in the Heston model, the solution of the PDE at $v=0$ satisfies the boundary condition that is obtained by inserting $v=0$ into (3.3); this is also referred to as a degenerated boundary condition:

$$
\frac{\partial u}{\partial \tau}=\kappa \eta \frac{\partial u}{\partial v}+\left(r^{\mathrm{d}}-r^{\mathrm{f}}\right) s \frac{\partial u}{\partial s}-r^{\mathrm{d}} u .
$$

In traditional literature (see, for example, Tavella and Randall 2000), the maximum variance boundary for call options is imposed as $u\left(s, V_{\max }, r^{\mathrm{d}}, \tau\right)=s$, but experiments show that this introduces a boundary layer. In combination with the PDE becoming convection-dominated around $v \approx 0$, this can result in oscillations if no upwinding is applied. To prevent this problem but still use central schemes, the option value at the maximum variance boundary is assumed to satisfy

$$
\frac{\partial^{2} u\left(s, V_{\max }, r^{\mathrm{d}}, \tau\right)}{\partial v^{2}}=0
$$

For the interest rate dimension, which is needed in the Heston Hull-White model, the boundary conditions are taken as

$$
\frac{\partial u\left(s, v, \pm R_{\max }^{\mathrm{d}}, \tau\right)}{\partial r^{\mathrm{d}}}=0 .
$$

Using the discretizations, boundary and initial condition, the following initial value problem for stiff ordinary differential equations (ODEs) is derived:

$$
\left.\begin{array}{rl}
\boldsymbol{u}^{\prime}(\tau) & =\boldsymbol{A} \boldsymbol{u}(\tau)+\boldsymbol{g}(\tau), \\
\boldsymbol{u}\left(\tau_{0}\right) & =\phi(\boldsymbol{s}(T)),
\end{array}\right\}
$$

where $\boldsymbol{u}(\tau)$ denotes the vector of discrete solutions $\boldsymbol{u}_{i, j, k}(\tau):=u\left(s_{i}, v_{j}, r_{k}^{\mathrm{d}}, \tau\right)$ ordered lexicographically, $\boldsymbol{g}(\tau)$ is a vector determined by the boundary conditions and $s(T)$ denotes the grid in the $s$-direction at maturity.

\subsubsection{Time discretization}

As the Heston model is a two-dimensional problem in space, the ODEs also have two space dimensions. To solve problems with dimensions higher than one, splitting techniques are relevant.

The splitting scheme used in this research is the Hundsdorfer-Verwer scheme. For more details, we refer to Hundsdorfer and Verwer (2003) for the derivation of the scheme, and to In't Hout and Welfert (2009) for a more detailed explanation of the alternating direction implicit (ADI) schemes in this context. 


\subsection{Computing CVA and its sensitivities}

To estimate CVA, we need $\operatorname{EE}(t)$ at any time $t \in\left[t_{0}, T\right]$ during the life of the derivative. Next to that, we need the probability of default at any time. Following Gregory (2010), we define $q_{l}=q\left(t_{l-1}, t_{l}\right)$ as the probability that the counterparty will default in the interval $\left[t_{l}-\mathrm{d} t, t_{l}\right]$. Using the so-called hazard rate $\lambda_{\text {haz }}$, the survival probability $P_{\text {surv }}(t)$ is defined as

$$
P_{\text {surv }}(t):=\mathrm{e}^{-\lambda_{\text {haz }} t} .
$$

Using this definition, we can derive the probability to default in interval $(t-\mathrm{d} t, t)$ conditioned on no prior default, as follows:

$$
q(t-\mathrm{d} t, t)=P_{\text {surv }}(t)-P_{\text {surv }}(t-\mathrm{d} t) .
$$

For any counterparty for which a credit default swap (CDS) is available for protection, this entity can be calculated from the CDS spread. As shown in Whetten et al (2004), the annual premium payment $c$ of a CDS can be calculated as

$$
c=\frac{(1-\delta) \sum_{l=1}^{N} P\left(t_{0}, t_{l}\right)\left(q_{l-1}-q_{l}\right)}{\sum_{l=1}^{N} P\left(t_{0}, t_{l}\right) q_{l} \mathrm{~d} t+\sum_{l=1}^{N} P\left(t_{0}, t_{l}\right)\left(q_{l-1}-q_{l}\right)(\mathrm{d} t / 2)},
$$

where $\mathrm{d} t$ denotes the payment interval. In this research, we assume annual premiums of 400 basis points (bps), which correspond to a hazard rate of $6.6 \times 10^{-2}$. Now, in a discrete setting, CVA can be calculated as

$$
\mathrm{CVA}=(1-\delta) \sum_{l=1}^{N} q\left(t_{l-1}, t_{l}\right) \mathrm{EE}^{*}\left(t_{l}\right)
$$

By using this expression, the first and second derivative of the CVA with respect to $\Theta_{0}$ (where $\Theta_{0}$ can be $S_{0}, V_{0}$ and $R_{0}^{\mathrm{d}}$ ) can be derived as follows:

$$
\begin{aligned}
\frac{\partial \mathrm{CVA}}{\partial \Theta_{0}} & =\frac{\partial}{\partial \Theta_{0}}(1-\delta) \sum_{l=1}^{N} q\left(t_{l-1}, t_{l}\right) \mathrm{EE}^{*}\left(t_{l}\right), \\
& =(1-\delta) \sum_{l=1}^{N} q\left(t_{l-1}, t_{l}\right) \frac{\partial \mathrm{EE}^{*}\left(t_{l}\right)}{\partial \Theta_{0}},
\end{aligned}
$$

where, in the second equality, we assume independence between the default probability and $\Theta_{0}$ as well as between the recovery rate $\delta$ and $\Theta_{0}$. Note that the assumption of independence between the default probability and $\Theta_{0}$ can be relaxed by modeling the default probability as a stochastic process that depends on $\Theta_{0}$, as is done in Hull 
and White (2012). ${ }^{2}$ Similarly, for the second derivative, we have

$$
\frac{\partial^{2} \mathrm{CVA}}{\partial \Theta_{0}^{2}}=(1-\delta) \sum_{l=1}^{N} q\left(t_{l-1}, t_{l}\right) \frac{\partial^{2} \mathrm{EE}^{*}\left(t_{l}\right)}{\partial \Theta_{0}^{2}} .
$$

To compute $\partial \mathrm{EE}^{*}(t) / \partial \Theta_{0}$ in (3.14), first, the derivative is rewritten as follows:

$$
\frac{\partial \mathrm{EE}^{*}(t)}{\partial \Theta_{0}}=\frac{\partial \mathrm{EE}^{*}(t)}{\partial S_{t}} \frac{\partial S_{t}}{\partial \Theta_{0}}+\frac{\partial \mathrm{EE}^{*}(t)}{\partial V_{t}} \frac{\partial V_{t}}{\partial \Theta_{0}}+\frac{\partial \mathrm{EE}^{*}(t)}{\partial R_{t}^{\mathrm{d}}} \frac{\partial R_{t}^{\mathrm{d}}}{\partial \Theta_{0}} .
$$

At every intermediate time point $t_{l}$, the finite difference method stores the prices for the entire grid in the vector $\boldsymbol{u}^{l}=\mathrm{EE}^{*}\left(t_{l}\right)$. On this grid, we can approximate $\partial \mathrm{EE}^{*}\left(t_{l}\right) / \partial \Theta_{t}$ and $\partial^{2} \mathrm{EE}^{*}\left(t_{l}\right) / \partial \Theta_{t}^{2}$ by multiplying with the difference matrixes $\boldsymbol{A}_{\Theta_{t}}(t)$ and $\boldsymbol{A}_{\Theta_{t}^{2}}(t)$. These are defined as follows: ${ }^{3}$

$$
\begin{gathered}
\frac{\partial \mathrm{EE}^{*}\left(t_{l}\right)}{\partial \Theta_{t}} \approx \boldsymbol{A}_{\Theta_{t}}\left(t_{l}\right) \boldsymbol{u}^{l}=\frac{\partial \boldsymbol{u}\left(t_{l}\right)}{\partial \Theta_{t}}+\mathcal{O}\left(\Delta \Theta_{t}^{2}\right), \\
\frac{\partial^{2} \mathrm{EE}^{*}\left(t_{l}\right)}{\partial \Theta_{t}^{2}} \approx \boldsymbol{A}_{\Theta_{t}^{2}}\left(t_{l}\right) \boldsymbol{u}^{l}=\frac{\partial^{2} \boldsymbol{u}\left(t_{l}\right)}{\partial \Theta_{t}^{2}}+\mathcal{O}\left(\Delta \Theta_{t}^{2}\right) .
\end{gathered}
$$

So, the partial derivatives of the exposure in (3.16) are obtained from the finite difference grid. The partial derivatives of the state variables with respect to the initial conditions are analyzed in the following subsections for all the possible choices of $\Theta$.

\subsubsection{Sensitivity with respect to initial FX rate}

To compute the sensitivity with respect to the initial underlying FX rate $\left(\Theta_{0}=S_{0}\right)$, we first note that the future variance and future short rate are independent of $S_{0}$, such that $\partial V_{t} / \partial S_{0}=\partial R_{t}^{\mathrm{d}} / \partial S_{0}=0$. However, $\partial S_{t} / \partial S_{0}$ is clearly nonzero, and this can be computed by the pathwise Monte Carlo method. Because $S_{0}$ follows a geometric Brownian motion (GBM) in the Heston Hull-White model, we can assume

$$
S_{t}=S_{0} \mathrm{e}^{\left(R_{t}^{\mathrm{d}}-r^{f}-\left(V_{t} / 2\right)\right) t+\sqrt{V_{t}} \sqrt{t} Z},
$$

where $Z$ is a standard normal random variable. Consequently, following Broadie and Glasserman (1996), for the first and second derivative, we have

$$
\begin{aligned}
\frac{\partial S_{t}}{\partial S_{0}} & =\mathrm{e}^{\left(R_{t}^{\mathrm{d}}-r^{f}-V_{t} / 2\right) t+\sqrt{V_{t}} \sqrt{t} Z}=\frac{S_{t}}{S_{0}}, \\
\frac{\partial^{2} S_{t}}{\partial S_{0}^{2}} & =0 .
\end{aligned}
$$

\footnotetext{
${ }^{2}$ In this specific case, (3.14) will have an extra term, but the sensitivities can still be computed. We leave this application for future work.

${ }^{3}$ Note that for $\Theta_{t}=r_{t}^{\mathrm{d}}, \boldsymbol{A}_{r}(t)$ is a time-dependent matrix; as in the case of the stochastic interest rate, the drift can be time dependent because of the yield curve (see, for example, the Hull-White model).
} 
Now, at any time point $t_{l}$, both partial derivatives from (3.16) can be computed for every path, such that $\partial \mathrm{EE}^{*}(t) / \partial S_{0}$ is obtained by averaging.

To compute $\partial^{2} \mathrm{CVA} / \partial S_{0}^{2}$, we need $\partial^{2} \mathrm{EE}^{*}(t) / \partial S_{0}^{2}$; this yields

$$
\begin{aligned}
\frac{\partial^{2} \mathrm{EE}^{*}(t)}{\partial S_{0}^{2}} & =\frac{\partial}{\partial S_{0}}\left(\frac{\partial \mathrm{EE}^{*}(t)}{\partial S_{t}} \frac{\partial S_{t}}{\partial S_{0}}\right) \\
& =\left(\frac{\partial}{\partial S_{0}} \frac{\partial \mathrm{EE}^{*}(t)}{\partial S_{t}}\right) \frac{\partial S_{t}}{\partial S_{0}}+\frac{\partial \mathrm{EE}^{*}(t)}{\partial S_{t}}\left(\frac{\partial}{\partial S_{0}} \frac{\partial S_{t}}{\partial S_{0}}\right) \\
& =\left(\frac{\partial^{2} \mathrm{EE}^{*}(t)}{\partial S_{t}^{2}} \frac{\partial S_{t}}{\partial S_{0}}\right) \frac{\partial S_{t}}{\partial S_{0}}+\frac{\partial \mathrm{EE}^{*}(t)}{\partial S_{t}} \frac{\partial^{2} S_{t}}{\partial S_{0}^{2}} \\
& =\frac{\partial^{2} \mathrm{EE}^{*}(t)}{\partial S_{t}^{2}}\left(\frac{S_{t}}{S_{0}}\right)^{2},
\end{aligned}
$$

where the second derivative of EE with respect to $S_{t}$ can be obtained from (3.18), and $\left(S_{t} / S_{0}\right)^{2}$ can be obtained from the scenario generation.

\subsubsection{Sensitivity with respect to initial variance}

In the case of sensitivity with respect to initial variance $\left(\Theta_{0}=V_{0}\right)$, the future short rate is independent of $V_{0}$, such that (3.16) can be simplified to

$$
\frac{\partial \mathrm{EE}^{*}(t)}{\partial V_{0}}=\frac{\partial \mathrm{EE}^{*}}{\partial S_{t}} \frac{\partial S_{t}}{\partial V_{0}}+\frac{\partial \mathrm{EE}^{*}}{\partial V_{t}} \frac{\partial V_{t}}{\partial V_{0}} .
$$

The partial derivatives of the exposures can be extracted from the finite difference grid by (3.17), such that only $\partial S_{t} / \partial V_{0}$ and $\partial V_{t} / \partial V_{0}$ are unknown. In the Heston model, the variance is modeled by a square root process, which has its difficulties. In this model, the variance process can and will reach zero, such that a straightforward derivative of (3.19) with respect to $V_{0}$ is not defined for every path at every time. This also holds for $\partial V_{t} / \partial V_{0}$, because when the discretized SDE of the variance process is differentiated with respect to $V_{0}$, the square root will appear in the denominator, which makes the derivative intractable. Further, in Chan and Joshi (2010) it is noted that the sensitivities of the variance process with respect to initial inputs can grow very quickly and potentially blow up. We therefore approximate these partial derivatives by a local bump-and-revalue approach, as follows:

$$
\begin{aligned}
& \frac{\partial S_{t}}{\partial V_{0}} \approx \frac{\tilde{S}_{m}-S_{m}}{\epsilon_{v}}, \\
& \frac{\partial V_{t}}{\partial V_{0}} \approx \frac{\tilde{V}_{m}-V_{m}}{\epsilon_{v}},
\end{aligned}
$$

where $\tilde{S}$ and $\tilde{V}$ are modeled with $V_{0}+\epsilon_{v}$ as the initial variance. Note that when an analytic expression for these partial derivatives is available, the method will gain in efficiency. 


\subsubsection{Sensitivity with respect to initial domestic interest rate}

To measure the sensitivity with respect to the initial domestic short rate $R_{0}^{\mathrm{d}}$, (3.16) is simplified to

$$
\frac{\partial \mathrm{EE}^{*}(t)}{\partial r_{0}^{\mathrm{d}}}=\frac{\partial \mathrm{EE}^{*}}{\partial S_{t}} \frac{\partial S_{t}}{\partial R_{0}^{\mathrm{d}}}+\frac{\partial \mathrm{EE}^{*}}{\partial R_{t}^{\mathrm{d}}} \frac{\partial R_{t}^{\mathrm{d}}}{\partial R_{0}^{\mathrm{d}}},
$$

where $\partial V_{t} / \partial R_{0}^{\mathrm{d}}$ is 0 because the future variance is independent of the initial short rate. Similar to the previous cases, the partial derivatives of EE with respect to $S_{t}$ and $R_{t}^{\mathrm{d}}$ can be derived from the finite difference grid by (3.17).

The partial derivatives of the state variables with respect to $R_{0}^{\mathrm{d}}$ can be computed along the path in the Monte Carlo simulation. For any discrete time point $t_{l}(0<l<$ $N)$, we can create a recursive formula for $\partial S_{t_{l}} / \partial R_{0}^{\mathrm{d}}$.

$$
\frac{\partial S_{t_{l}}}{\partial R_{0}^{\mathrm{d}}}=\frac{\partial S_{t_{l}}}{\partial S_{t_{l-1}}} \frac{\partial S_{t_{l-1}}}{\partial R_{0}^{\mathrm{d}}}+\frac{\partial S_{t_{l}}}{\partial R_{t_{l-1}}} \frac{\partial R_{t_{l-1}}^{\mathrm{d}}}{\partial R_{0}^{\mathrm{d}}}
$$

where (when $S_{t}$ is driven by a GBM) we have

$$
\begin{aligned}
\frac{\partial S_{t_{l}}}{\partial S_{t_{l-1}}} & =\frac{S_{t_{l}}}{S_{t_{l-1}}}, \\
\frac{\partial S_{t_{l}}}{\partial R_{t_{l-1}}^{\mathrm{d}}} & =S_{t_{l}} \Delta t .
\end{aligned}
$$

Here, $\Delta t=t_{l}-t_{l-1}$ is the uniform time increment in one time step. The interest rate is modeled by the Hull-White model (Hull and White 1993), such that a Euler scheme as a discretization yields

$$
R_{t_{l+1}}^{\mathrm{d}}=R_{t_{l}}^{\mathrm{d}}+\lambda\left[\theta\left(t_{l}\right)-R_{t_{l}}^{\mathrm{d}}\right] \Delta t+\gamma \sqrt{\Delta t} Z_{t_{l}},
$$

where $Z \sim N(0,1)$. From this, we can recursively derive

$$
\frac{\partial R_{t_{l-1}}}{\partial R_{0}^{\mathrm{d}}}=(1-\lambda \Delta t)^{(l-1)} .
$$

The first time step gives us the initial condition

$$
\frac{\partial S_{t_{1}}}{\partial R_{0}^{\mathrm{d}}}=S_{0} \Delta t
$$

Using this recursive formula, together with the finite difference approximations, we can estimate the sensitivity with respect to $R_{0}^{\mathrm{d}}$ at any time point, without the need of an extra Monte Carlo simulation.

Similar to the case for EE, the computation of the first and second derivatives with respect to initial underlying states can be summarized as follows: 
- generating scenarios/paths by Monte Carlo simulation;

- at each time point $t_{l}$, for the entire grid, calculate option sensitivities $\partial \mathrm{EE}^{*}\left(t_{l}\right) / \partial \Theta$ and $\partial^{2} \mathrm{EE}^{*}\left(t_{l}\right) / \partial \Theta^{2}$; for barrier options, check if the option is not exercised $\left(S_{t^{*}}<B\right.$ for all $\left.t^{*} \leqslant t_{l}\right)$;

- set the first and second derivatives at each path as the calculated sensitivities if the option is not exercised; otherwise, set them equal to 0 ;

- compute the empirical distribution of the sensitivities at each exercise time;

- calculate $\partial \mathrm{EE}^{*}\left(t_{l}\right) / \partial \Theta$ and $\partial^{2} \mathrm{EE}^{*}\left(t_{l}\right) / \partial \Theta^{2}$ by averaging.

\subsection{Pricing a portfolio}

In this research, the finite difference grid is used to price multiple options with different strikes and maturities in one sweep on one grid. The portfolios considered here are constructed of European options and a first-order exotic barrier option. The value $\Pi$ of a portfolio of $N$ options can be seen as the sum of the option prices

$$
\Pi(t)=\sum_{i=1}^{N} U_{i}\left(S_{t}, K_{i}, T_{i}\right),
$$

where $K_{i}$ is the strike, $T_{i}$ is the maturity and $U_{i}$ is the price of option $i$. In this paper, option $i$ can thus be a European call or put option, or a barrier option. We assume that all options in the portfolio can be netted.

Together with the Monte Carlo scenario generation, this gives us the exposure profile of the sum of the option values at any future time point. The duration of the portfolio is equal to the longest maturity in the portfolio:

$$
\tilde{T}=\max _{i \in[1, N]} T_{i}
$$

Again, at this maximum maturity, all the option prices on the grid are known; therefore, the time is reversed such that the payoff formula (3.35) can be used as an initial condition that equals the sum of all individual payoff functions belonging to options with maturity equal to the maximum maturity $\tilde{T}$ :

$$
\phi_{P}\left(S_{t}\right)=\sum_{i=1}^{N} \phi_{i}\left(S_{t}, K_{i}, T_{i}\right) \mathbf{1}_{\left\{T_{i}=\tilde{T}\right\}} .
$$

Important in the context of this research is that the option-specific characteristics are only introduced by the initial condition and the boundary conditions. Because the portfolio consists of a sum of options, the boundary conditions for the portfolio will also be just a sum of these limiting conditions, such that our time-stepping routine in the finite difference procedure can be updated as follows. 
- From $\boldsymbol{u}^{l}$, calculate $\tilde{\boldsymbol{u}}^{l-1}$ by the ADI splitting scheme.

- Update the portfolio value with possible other option values:

$$
\boldsymbol{u}^{l-1}=\tilde{\boldsymbol{u}}^{l-1}+\sum_{i=1}^{N} \phi_{i}\left(\boldsymbol{s}, K_{i}, T_{i}\right) \mathbf{1}_{\left\{T_{i}=t_{l-1}\right\}},
$$

where $s$ is a vector of the same size as $\boldsymbol{u}$, consisting of all $s$ grid points.

- Update all boundary conditions.

- If $l>0$, repeat the procedure.

By applying this time-stepping procedure, $\boldsymbol{u}^{0}$ will be the value of the portfolio at time $t=t_{0}$. For the computation of exposure of a portfolio over time, options that are not path-dependent can be included on one grid. By using only one grid, there will be no extra computational time for these extra options.

In the case of a portfolio of a call, put and barrier considered in this research, the EEs of the call and put options are computed using only one grid, whereas the EE of the barrier option is computed using the algorithm from Section 3.1. In general, for the computation of path-dependent options, first a separate finite difference procedure needs to be done. Next, while computing this individual options exposure, it needs to be checked, for any scenario, if the simulated scenario should be exercised or not. At every time step, the portfolio exposure is then computed as the sum of the individual barrier exposure and the call and put option exposure. To summarize, the computational time of computing exposure for a portfolio is determined by

- one Monte Carlo simulation to compute all the scenarios,

- one finite difference procedure to compute the price grid for all non-pathdependent options,

- a separate finite difference procedure per path-dependent option.

Note that the same holds for path-dependent American options.

\section{NUMERICAL RESULTS}

The numerical results are divided into two parts. First, we present our numerical study on the accuracy and convergence under the two-dimensional Heston model. In this case, all results are validated by the semi-analytical COS method.

Second, we further assess the accuracy of the interpolation schemes by looking into the numerically challenging Heston Hull-White model, where the number of grid points per dimension is smaller, which can cause a larger interpolation error. We do 
TABLE 2 Model parameters for various test cases.

\begin{tabular}{llc}
\hline & Case A & Case B \\
\hline Spot $\left(S_{0}\right)$ & 1.364 & 138.1 \\
Foreign short rate $\left(r^{f}\right)$ & 0.01 & 0.10 \\
Initial variance $\left(V_{0}\right)$ & 0.029 & 0.029 \\
Mean reversion speed of variance $(\kappa)$ & 4.42 & 1.50 \\
Mean reversion variance level $(\eta)$ & 0.0240 & 0.0707 \\
Volatility of volatility $(\sigma)$ & 0.46 & 0.63 \\
Initial domestic short rate $\left(R_{0}^{\mathrm{d}}\right)$ & 0.01 & 0.03 \\
Mean reversion speed of interest rate $(\lambda)$ & 0.5 & 0.5 \\
Mean reversion interest rate $(\theta)$ & 0.05 & 0.05 \\
Volatility of short rate $(\gamma)$ & 0.02 & 0.02 \\
$s, v$-correlation $\left(\rho_{1,2}\right)$ & -0.45 & -0.76 \\
$s, r^{\mathrm{d}}$-correlation $\left(\rho_{1,3}\right)$ & 0.501 & -0.011 \\
$v, r^{\mathrm{d}}$-correlation $\left(\rho_{2,3}\right)$ & -0.96 & -0.96 \\
Maturity $(T)$ & 0.5 & 1.0 \\
Strike $(K)$ & 1.360 & 138.1 \\
Barrier $(B)$ & 1.20 & 120 \\
\hline
\end{tabular}

this in combination with the EE computation of OT options, which are discontinuous during the entire lifetime of the option. ${ }^{4}$ Next to that, we show the sensitivities with respect to $V_{0}$ and $R_{0}^{\mathrm{d}}$.

The parameters are chosen according to Table 2 . In case A, the foreign interest rate is equal to the initial domestic rate; next to that, the option is out-of-the-money (OTM) at inception. The level of the initial FX rate is set to 1.3639 , which is a real market-quoted EUR/USD FX rate from June 2014, whereas the other parameters satisfy characteristics observed in literature, such as negative correlation, low volatility and interest rates and small maturities (see, for example, Schoutens et al 2004 and Albrecher et al 2007). In this test, the well-known Feller condition is satisfied. In case B, the initial FX rate is set to 138.1, a real EUR/JPY FX rate from June 2014; the option is at-the-money (ATM) at inception, while the initial domestic interest rate is higher than the foreign interest rate. In this case, the other model parameters are chosen such that the Feller condition is violated. ${ }^{5}$

\footnotetext{
${ }^{4}$ We would like to thank an anonymous reviewer for this suggestion.

${ }^{5}$ When the Feller condition is not satisfied, the variance process can become zero, and numerical methods can become unstable.
} 


\subsection{Heston model}

In the case of the Heston model, the domestic interest rate $R_{t}^{\mathrm{d}}$ is assumed to be constant over time, such that

$$
D(0, t)=\mathrm{e}^{-\int_{t_{0}}^{t} R_{\xi}^{\mathrm{d}} \mathrm{d} \xi}=\mathrm{e}^{-r^{\mathrm{d}} t} .
$$

Further, in the pricing $\operatorname{PDE}$ (3.3), $\lambda$ and $\gamma$ are assumed to be 0 .

\subsubsection{Single barrier options: numerical setup}

Computing the exposure of barrier options is more challenging than computing the exposure of European options. Barrier options are path-dependent and have a discontinuous initial condition. It is this discontinuous nature of the payoff function in particular that may complicate the accurate estimation of sensitivities, especially for higher-order ones. Because we have a benchmark solution for down-and-out put (DOP) options, we do an extensive error analysis for this option type, but the method can also be applied to down-and-out call (DOC), up-and-out put (UOP) and up-and-out call (UOC) options, and all the other "in" (instead of "out") variants.

The computed EE, $\mathrm{PFE}_{2.5 \%}$ and $\mathrm{PFE}_{97.5 \%}$ are shown in Figures 2(a) and 2(b). The starting level of the EE equals the option price at $t=t_{0}$ and shows a small increase toward maturity. The PFE, however, shows more interesting behavior. Starting at the option price, the PFE is increasing over time and shows a steep growth close to maturity. Intuitively, the increase of the higher quantile makes sense; when moving $t^{*} \in\left[t_{0}, T\right]$ closer to maturity, the hitting probability conditioned on no prior barrier hit will become smaller, such that for in-the-money paths the price will resemble a straightforward European option value more and more. The mean (EE) is not heavily affected, because the probability of the barrier being hit up to time $t^{*}$ is increased, which will lower the option value.

\subsubsection{Accuracy and convergence}

As a benchmark, the COS method can be applied to evaluate barrier options accurately and efficiently. For details on the pricing procedure using this Fourier cosine method, we refer to Fang and Oosterlee (2011). Here, we use this efficient pricing technique by computing prices for an entire grid of possible market scenarios. Similar to the grid used for the finite difference procedure, this grid is chosen so that it is large enough to contain all future market scenarios generated by the Monte Carlo scenario generation. We choose 500 points in the $s$-direction and 300 points in the $v$-direction, and both are densely distributed around the expected means. Prices for all the scenarios are obtained by a spline interpolation on the COS grid.

For the sensitivities with respect to $S_{0}$, we run this procedure two (in the case of Delta) or three (in the case of Gamma) times, with an initial condition bumped by 
FIGURE 2 Exposures (EE, $\mathrm{PFE}_{2.5 \%}$ and $\mathrm{PFE}_{97.5 \%}$ ) and the first and second derivative profiles over time under the Heston dynamics for tests A and B.
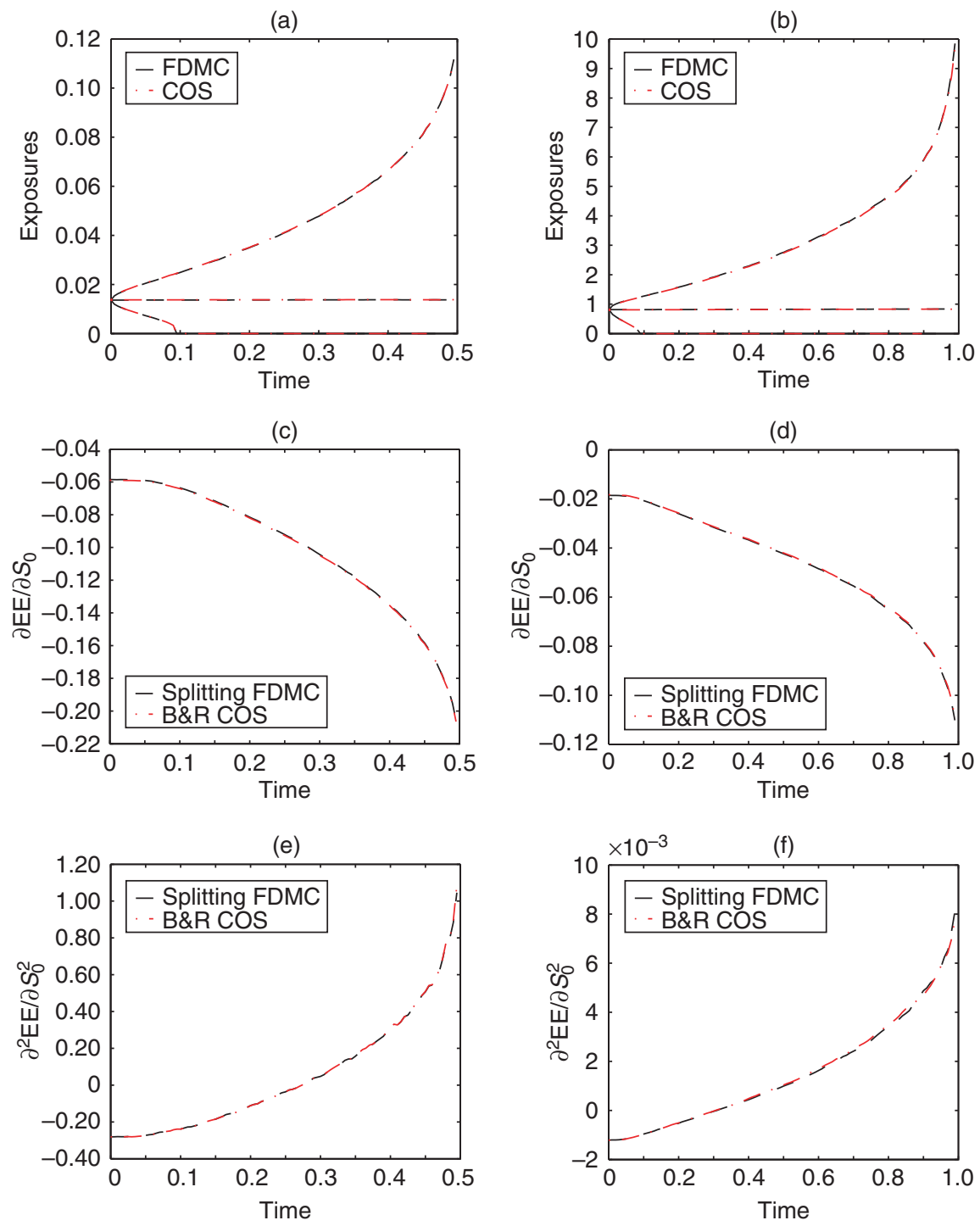

The dashed black line is computed using the FDMC method, whereas the dashed red line is computed using the COS method. In the case of the sensitivities, the results corresponding to the COS method are obtained using a bump-and-revalue (B\&R) procedure, whereas for the FDMC method the derivative is splitted, as explained in Section 3.3. (a) Exposure profiles for test A. (b) Exposure profiles for test B. (c) Delta profile for test A. (d) Delta profile for test B. (e) Gamma profile for test A. (f) Gamma profile for test B. 
TABLE 3 Relative $L_{2}$ and $L_{\infty}$ errors compared with the COS method using the linear or spline interpolation.

\begin{tabular}{cccccc}
\hline \multirow{2}{*}{ Error } & Quantity & Test A & Test B & Test A & Test B \\
\hline$\|\cdot\|_{\infty}$ & $\mathrm{EE}$ & $2.1728 \times 10^{-3}$ & $3.7094 \times 10^{-3}$ & $2.1690 \times 10^{-3}$ & $3.7006 \times 10^{-3}$ \\
& $\mathrm{PFE}_{97.5 \%}$ & $4.9120 \times 10^{-3}$ & $5.3274 \times 10^{-3}$ & $4.9213 \times 10^{-3}$ & $5.2280 \times 10^{-3}$ \\
& $\frac{\partial \mathrm{EE}}{\partial S_{0}}$ & $4.7905 \times 10^{-3}$ & $6.6173 \times 10^{-3}$ & $4.8058 \times 10^{-3}$ & $6.6243 \times 10^{-3}$ \\
& $\frac{\partial^{2} \mathrm{EE}}{\partial S_{0}^{2}}$ & $3.5990 \times 10^{-2}$ & $3.7086 \times 10^{-2}$ & $3.5982 \times 10^{-2}$ & $3.7107 \times 10^{-2}$ \\
$\|\cdot\|_{2}$ & $\begin{array}{c}\mathrm{EE} \\
\mathrm{PFE}\end{array}$ & $1.8225 \times 10^{-3}$ & $3.1294 \times 10^{-3}$ & $1.8197 \times 10^{-3}$ & $3.1216 \times 10^{-3}$ \\
& $\frac{\partial \mathrm{EE}}{\partial S_{0}}$ & $3.0751 \times 10^{-3}$ & $5.2244 \times 10^{-3}$ & $3.0832 \times 10^{-3}$ & $5.2280 \times 10^{-3}$ \\
& $\frac{\partial^{2} \mathrm{EE}}{\partial S_{0}^{2}}$ & $2.5988 \times 10^{-2}$ & $2.2843 \times 10^{-2}$ & $2.5921 \times 10^{-2}$ & $2.2881 \times 10^{-2}$ \\
& & & & & \\
\hline
\end{tabular}

$\epsilon_{s}$. From the resulting EEs, the sensitivities are computed using the following finite difference formulas:

$$
\begin{aligned}
\frac{\partial \mathrm{EE}(t)}{\partial S_{0}} & \approx \frac{\mathrm{EE}_{S_{0}+\epsilon_{S}}(t)-\mathrm{EE}_{S_{0}-\epsilon_{S}}(t)}{2 \epsilon_{s}}, \\
\frac{\partial^{2} \mathrm{EE}(t)}{\partial S_{0}^{2}} & \approx \frac{\mathrm{EE}_{S_{0}+\epsilon_{S}}(t)-2 \mathrm{EE}_{S_{0}}(t)+\mathrm{EE}_{S_{0}-\epsilon_{S}}(t)}{\epsilon_{s}^{2}} .
\end{aligned}
$$

Figures 2(a) to 2(f) show that the exposure profiles and sensitivities over time computed with the FDMC method resemble the results computed by the Monte Carlo-COS method.

For an EE computed over $N_{T}$ evaluation dates, the relative $L_{2}$ and $L_{\infty}$ errors are computed as

$$
\begin{aligned}
\|\cdot\|_{\infty} & :=\frac{\max _{i=1, \ldots, N_{T}}\left|\mathrm{EE}_{i}^{\mathrm{COS}}-\mathrm{EE}_{i}^{\mathrm{FDMC}}\right|}{\max _{i=1, \ldots, N_{T}}\left|\mathrm{EE}_{i}^{\mathrm{COS}}\right|} \\
\|\cdot\|_{2} & :=\frac{\left(\sum_{i=1}^{N_{T}}\left(\mathrm{EE}_{i}^{\mathrm{COS}}-\mathrm{EE}_{i}^{\mathrm{FDMC}}\right)^{2}\right)^{1 / 2}}{\left(\sum_{i=1}^{N_{T}}\left(\mathrm{EE}_{i}^{\mathrm{COS}}\right)^{2}\right)^{1 / 2}}
\end{aligned}
$$

In Table 3, the errors between the FDMC method with 700 grid points in the $s$ direction and 350 in the $v$-direction are compared with the COS method. We can see that the relative error is below $1 \%$ in both $\mathrm{EE}$ and the first derivative. The second 
FIGURE 3 Error convergence of EE and first- and second-order sensitivities for tests A and B by increasing the number of grid points $(2 m \times m)$ used in the finite difference computation.

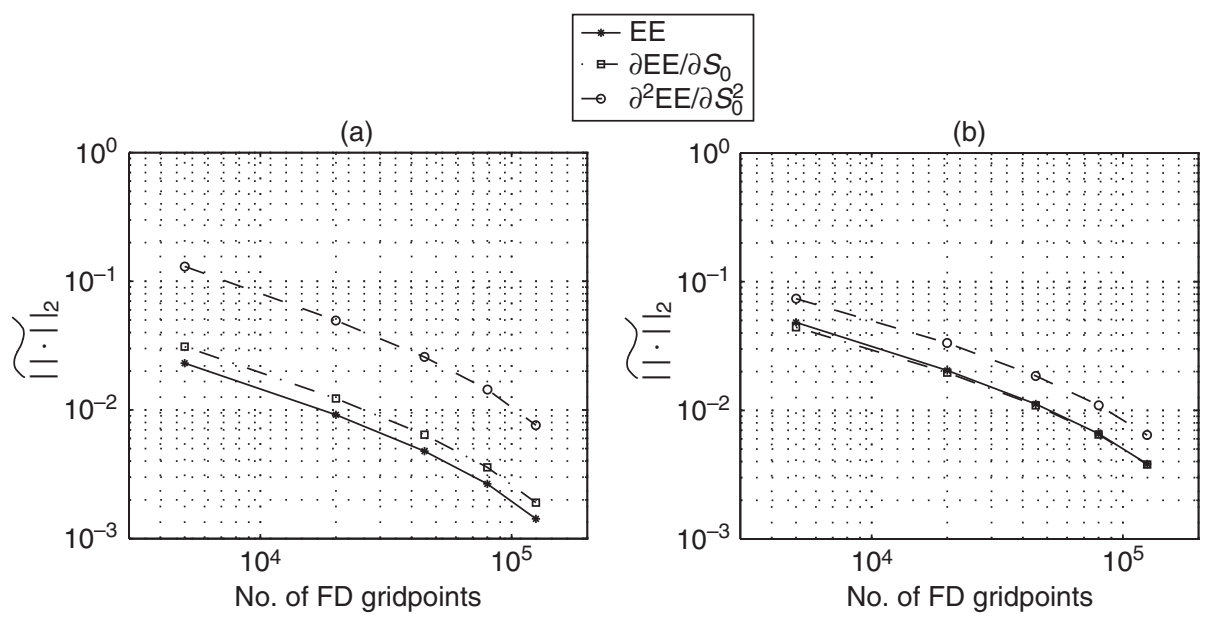

We use $m$ in the $v$-direction and $2 m$ in the $s$-direction. For every exposure computation, $\times 10^{5}$ paths are used, simulated with a fixed seed to avoid noise. Here, a spline interpolation is used, but similar analysis performed using a linear interpolation is shown in Table 3. (a) Test A. (b) Test B.

derivative, however, is accurate up to $5 \%$ in both the $L_{\infty}$ and $L_{2}$ norms. This is due to the fact that this absolute value of Gamma is already in the range of $10^{-4}$, such that the errors from the finite difference discretization have a larger impact. Further, we can see that the difference between a spline and linear interpolation is negligible.

The convergence with respect to the number of finite difference grid points is shown in Figures 3(a) and 3(b) for the EE and the first and second derivative with respect to $S_{0}$. In this case, the benchmark is the converged finite difference solution obtained with 700 and 350 points in the $s$ - and $v$-directions, respectively. The convergence is shown to be first-order in the number of grid points.

In Figures 4(a) and 4(b), we show the decline of the relative standard error (SE) in percentage of the mean by increasing the number of paths for tests A and B. Here, we computed the standard error using ten Monte Carlo simulations with different seeds. Typically, the Monte Carlo convergence is expected to be $1 / \sqrt{N_{p}}$, where $N_{p}$ is the number of Monte Carlo paths. We see that, for both tests, all quantities converge as expected.

Note that the computation time of the FDMC method heavily depends on the 
FIGURE 4 Convergence of the relative standard error (SE), Delta and Gamma of CVA for tests $A$ and $B$ for an increasing number of paths.

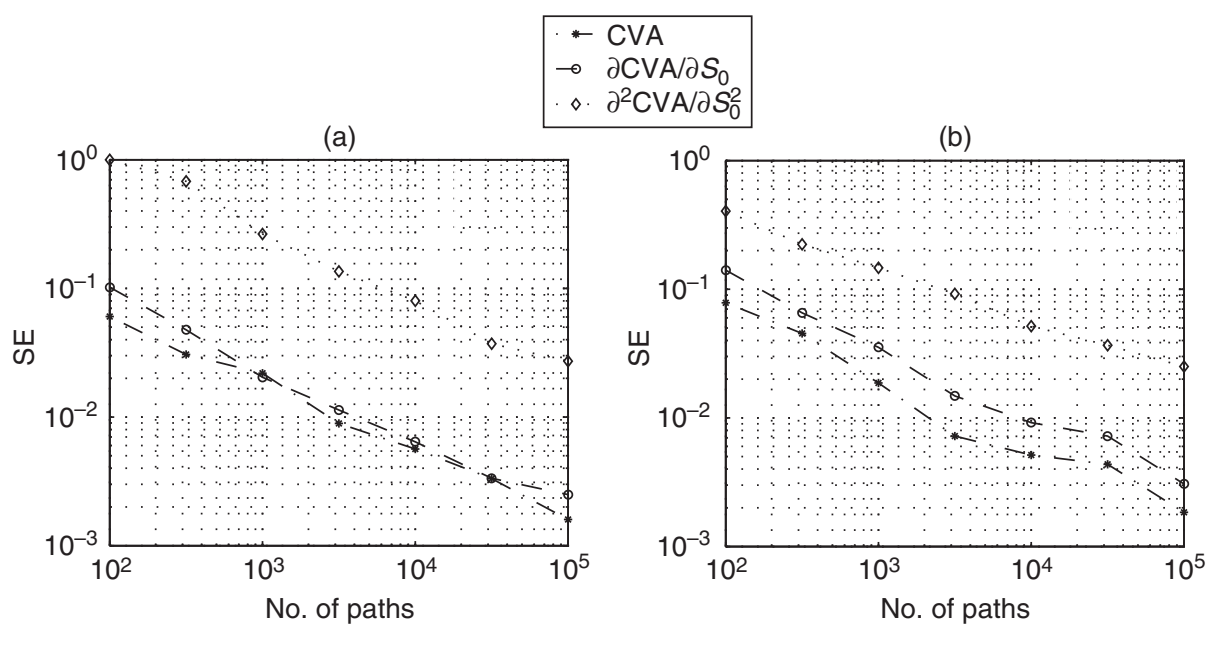

Here, the number of finite difference grid points is set equal to 350 in the $v$-direction and 700 in the $s$-direction, and the standard error is computed relative to the mean. (a) Test A. (b) Test B.

number of grid points. When we compare the computation time of the price grids computed by COS or FDMC, we see that the FDMC method is significantly faster. ${ }^{6}$

\subsubsection{Portfolio of options}

For the evaluation of CVA, we assume a recovery rate of $40 \%$. The hazard rate is computed by assuming a five-year CDS with a spread of 400bps paid quarterly. The euro discount factors are taken from April 2014, and the resulting survival probabilities up to one year are obtained as explained in Section 3.3. Because we assume the absence of wrong- and right-way risk, we can compute the CVA for any CDS spread. In this case, CVA is a linear function of the CDS spread.

Different options in one portfolio can have different strikes and maturities. Due to these different maturities, the finite difference procedure is faced with a discontinuity in time. To assess the possible effect on the accuracy, we consider a portfolio of two European options with different strike and maturity. We again compare the resulting exposure profiles and CVA values with the Monte Carlo COS method. For the benchmark, we compute separate exposure profiles for every option with the Monte Carlo COS method and compute the EE of the portfolios as the sum. This is similar for

${ }^{6}$ In our implementation, the COS grid was obtained by subsequently pricing every grid point, which probably can be optimized. 
TABLE 4 Tested portfolios.

\begin{tabular}{lllllc}
\hline & & Type & Maturity & Strike & Barrier \\
\hline \multirow{2}{*}{ Portfolio I } & Option 1 & Call & $T_{1}=1$ & $K_{1}=133$ & - \\
& Option 2 & Put & $T_{2}=0.4$ & $K_{2}=138$ & - \\
& Option 3 & Barrier & $T_{3}=0.8$ & $K_{3}=135$ & $B_{3}=120$ \\
\multirow{2}{*}{ Portfolio II } & Option 1 & Call & $T_{1}=1$ & $K_{1}=133$ & - \\
& Option 2 & Put & $T_{2}=0.4$ & $K_{2}=138$ & - \\
\hline
\end{tabular}

the sensitivities that are obtained by a bump-and-revalue procedure per option. In the FDMC method, the call and put options are computed simultaneously on one grid. The barrier option is computed on a separate grid, because for every path termination needs to be checked. The resulting option prices per path are added to the portfolio values, and from this the mean and quantiles can be calculated.

Again, we assume that the Heston dynamics drive the underlying risk factors. The Heston parameters that drive the underlying are chosen as in case B of the previous subsection. All the options in the portfolio are written on this single FX rate. We consider two portfolios: portfolio I consists of a call, put and barrier option, while portfolio II consists only of a call and put. Table 4 shows the option parameters for the two portfolios.

The results presented in this paper also hold for portfolios consisting of an arbitrary larger number of options, but for illustrative reasons we present results for only three options.

In Table 5, we show the CVA values. Here, we computed the CVA as a percentage of the portfolio value. The sensitivities are quoted relative to the sensitivities of the initial portfolio. This way, we can quantify the change between the CVA-adjusted and the non-CVA-adjusted portfolio:

$$
\begin{aligned}
\mathrm{CVA}_{\%} & :=100 \frac{\mathrm{CVA}}{\Pi}, \\
\Delta_{S_{0}} & :=100 \frac{\partial \mathrm{CVA}}{\partial S_{0}} / \frac{\partial \Pi}{\partial S_{0}}, \\
\Gamma_{S_{0}} & :=100 \frac{\partial^{2} \mathrm{CVA}}{\partial S_{0}^{2}} / \frac{\partial^{2} \Pi}{\partial S_{0}^{2}} .
\end{aligned}
$$

By looking at Figures 5(a) and 5(b), we can see that the EE drops at $t=0.4$, when the put option expires. This discontinuity is captured nicely by the FDMC method, where the put and call options are computed on one finite difference grid. By looking at Table 5, we can conclude that the resulting value adjustments are accurate compared 
TABLE 5 CVA, Delta and Gamma for the portfolios I and II as a percentage of non-adjusted values.

\begin{tabular}{lrrrr}
\hline & $\begin{array}{c}\text { Portfolio } \\
\text { I (\%) }\end{array}$ & $\begin{array}{c}\text { Portfolio } \\
\text { II (\%) }\end{array}$ & $\begin{array}{l}\text { Portfolio } \\
\text { I (\%) }\end{array}$ & $\begin{array}{l}\text { Portfolio } \\
\text { II (\%) }\end{array}$ \\
\hline CVA FDMC & 2.79 & 2.77 & 2.79 & 2.77 \\
CVA COS & 2.79 & 2.77 & 2.79 & 2.77 \\
$\Delta_{S_{0}}$ splitting FDMC & 23.49 & 18.78 & 23.29 & 18.72 \\
$\Delta_{S_{0}}$ B\&R COS & 23.52 & 18.85 & 23.40 & 18.79 \\
$\Gamma_{S_{0}}$ splitting FDMC & 2.58 & 2.42 & 2.57 & 2.41 \\
$\Gamma_{S_{0}}$ B\&R COS & 2.52 & 2.38 & 2.57 & 2.43 \\
\hline
\end{tabular}

The percentages are computed by spline and linear interpolation for the FDMC method and the benchmark COS method. The sensitivities in the COS method are obtained by a bump-and-revalue technique.

with the Monte Carlo COS method. Next to that, the difference between spline and linear interpolation is small.

In portfolio I, the call and put options have a bigger effect on the EE than the barrier option. Also, the higher PFE is heavily affected by the expiry of the put option. If we compare Figures 5(a) and 5(b), we can see that the impact of the expiring barrier option at $T=0.8$ is not reflected in the PFE, and it is only minor in the EE profile. This minor barrier effect is also visible when we compare the CVAs for portfolios I and II. The difference between these portfolios is due to the barrier option, and we can see a CVA difference of $1.5 \%$ in Table 5.

Next, if we look at the Delta profiles in Figures 5(c) and 5(d), we can see that for portfolio I the impact of the barrier option is reflected by a steep decrease at the expiry of the barrier option $T=0.8$. As this barrier option is absent in portfolio II, this decrease is absent in the Delta profile of portfolio II. This impact is also confirmed by looking at the sensitivity of CVA with respect to $S_{0}$ in Table 5, where the difference between portfolios I and II is in the region of $25 \%$ for $\Delta_{S_{0}}$.

In the case of Gamma, shown in Figures 5(e) and 5(f), the barrier option in portfolio I shows a steep increase at the expiry of the barrier option. The relative impact for Gamma, however, is smaller than for Delta. In Table 5, we see that the difference in Gamma between portfolios I and II is in the range of 3\%.

Further, we can see that the spline interpolation yields similar results to the linear interpolation. These results indicate that the effect of barrier options in a portfolio can be more severe in the sense of sensitivities than in CVA itself. Clearly, a small change in the EE profile can have a bigger impact on the first- and second-order sensitivity. Further, in the online appendix, we show that for barrier options the sensitivities are also more sensitive to changes in moneyness levels. 
FIGURE 5 Exposure, Delta and Gamma profiles for portfolios I and II over time for case $\mathrm{B}$, computed with the FDMC or COS method.

(a)

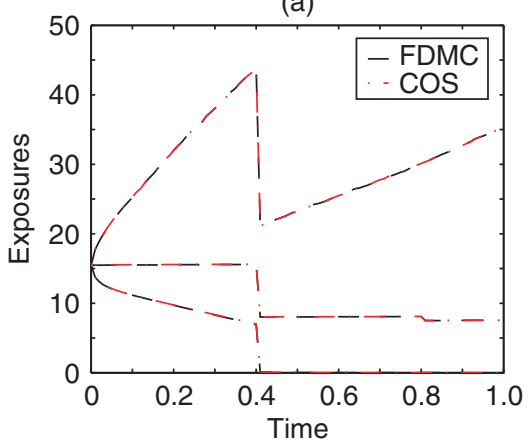

(c)

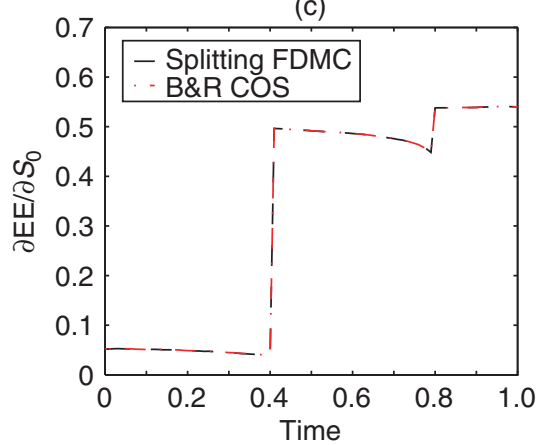

(e)

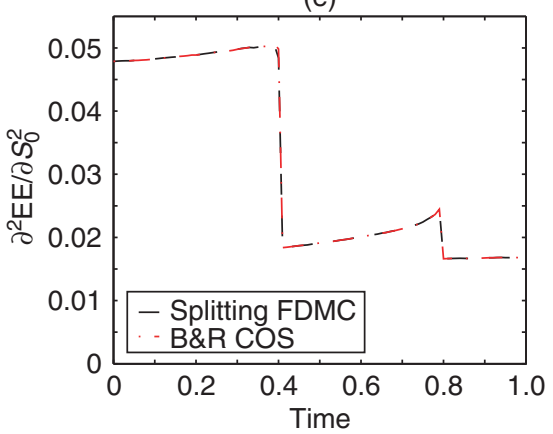

(b)

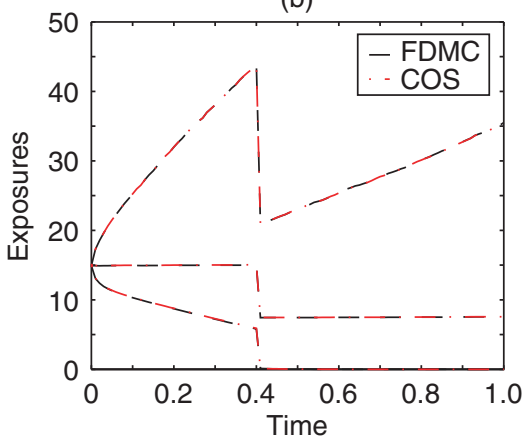

(d)

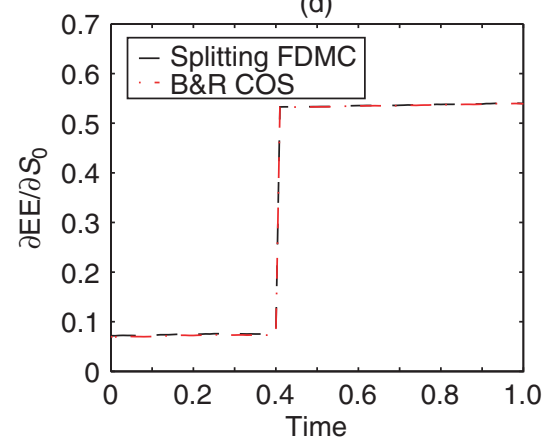

(f)

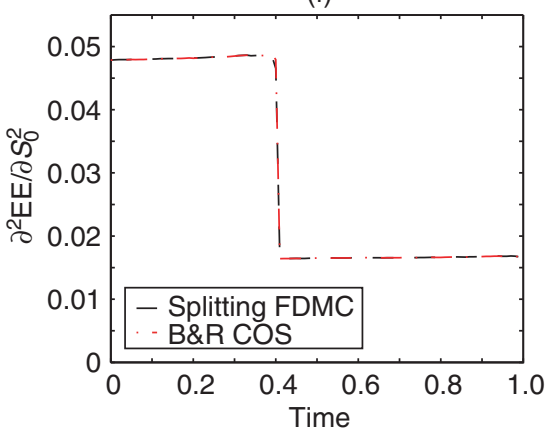

Again, the COS sensitivities are computed running two or three simulations from a bumped initial value. The results compared with the COS method are accurate up to an order of $\times 10^{-3}$. (a) Exposure profiles for portfolio I. (b) Exposure profiles for portfolio II. (c) Delta profile for portfolio I. (d) Delta profile for portfolio II. (e) Gamma profile for portfolio I. (f) Gamma profile for portfolio II. 
FIGURE 6 Exposures and PFE for a one-year OT option with barrier level $1.2 S_{0}$ and payout 100 .

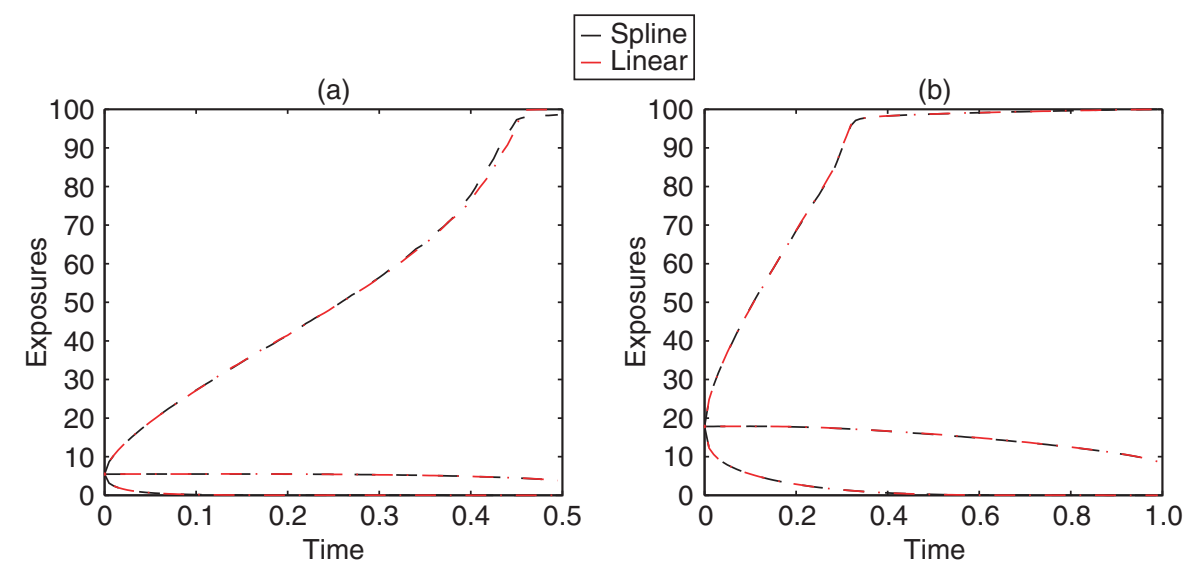

Here, we use 100 time points and the number of finite difference grid points is set equal to 100 in the $s$-direction and 50 in the $v$ - and $r^{\mathrm{d}}$-directions. (a) Test A. (b) Test B.

\subsection{Heston Hull-White model}

As the parameters are not calibrated in this research, the mean-reverting level in the short rate process $\theta(t)$ is assumed to be constant over time. ${ }^{7}$

\subsubsection{One-touch options}

The OT option only delivers a fixed payoff at maturity; therefore, it has a discontinuous profile during the entire lifetime of the option. Next to that, the three-dimensional dynamics of the Heston Hull-White model implies a coarser grid in every dimension. Because of this discontinuity and the coarser grid, the interpolation scheme is more important. In Figure 6, we show the exposures for tests A and B. We can see that the 97.5\% PFE in test $\mathrm{B}$ reaches the maximum payout level earlier than in test $\mathrm{A}$. The lower mean-reversion speed in combination with the higher volatility of volatility and long-term mean of the variance process in test B causes fatter tails, which imply a higher hitting probability.

The spline and linear exposures are very close. This is further shown in Table 6 , where we see that the $L_{\infty}$ and $L_{2}$ differences are in the range of $1 \%$.

\footnotetext{
${ }^{7}$ A study on real model impact on exposure, in which parameters are calibrated to real market data, is a subject for future study.
} 
TABLE 6 Relative $L_{2}$ and $L_{\infty}$ differences between exposure metrics, computed using linear and spline interpolation for one-year OT option, with barrier level $1.2 S_{0}$ and payout 100.

\begin{tabular}{llcc}
\hline Differences & Quantity & Test A & Test B \\
\hline$\|\cdot\|_{\infty}$ & EE & $5.889 \times 10^{-3}$ & $5.819 \times 10^{-3}$ \\
& PFE $_{97.5 \%}$ & $1.123 \times 10^{-2}$ & $6.310 \times 10^{-3}$ \\
& PFE $_{2.5 \%}$ & $4.327 \times 10^{-4}$ & $1.066 \times 10^{-3}$ \\
$\|\cdot\|_{2}$ & EE & $2.411 \times 10^{-3}$ & $3.002 \times 10^{-3}$ \\
& PFE $_{97.5 \%}$ & $6.454 \times 10^{-3}$ & $1.410 \times 10^{-3}$ \\
& PFE $_{2.5 \%}$ & $7.700 \times 10^{-4}$ & $1.504 \times 10^{-3}$ \\
\hline
\end{tabular}

\subsubsection{Other sensitivities}

Here, we look at Vega and Rho for OT options over time. Note that, in this case, the bump-and-revalue method uses two Monte Carlo simulations and estimates the derivative by

$$
\frac{\partial \mathrm{EE}(t)}{\partial \Theta} \approx \frac{\mathrm{EE}_{\Theta+\epsilon_{\Theta}}(t)-\mathrm{EE}_{\Theta}(t)}{\epsilon_{\Theta}}
$$

which is only first-order accurate in $\epsilon_{\Theta}$, which we choose as $0.01 \times V_{0}$ and $0.01 \times R_{0}^{\mathrm{d}}$, respectively. Figure 7 shows that the sensitivities computed by the splitting scheme and the bump-and-revalue method agree over time. This is further confirmed by looking at the relative differences that are again in the range of $1 \%$, as presented in Table 7.

\section{CONCLUSION}

In this research, we proposed a new computational technique to compute exposure profiles and their sensitivities. This paper extends the FDMC method described in de Graaf et al (2014) and is based on combining the Monte Carlo scenario generation with option valuation by solving a PDE on a grid. For every scenario at every time point, the option prices are obtained by interpolating the scenarios on this option grid. The EE needed for the computation of CVA is computed by averaging. For the Heston model, we have shown that, compared with a benchmark Monte Carlo COS method, the FDMC is a computationally efficient and accurate method; it can therefore serve as an alternative to the widely used American-style Monte Carlo approach, which in application to exotic options can suffer from regression bias.

The sensitivities with respect to $S_{0}$ and $R_{0}^{\mathrm{d}}$ are obtained efficiently by leveraging from the finite difference grid. Compared with a "brute force" bump-and-revalue technique, the sensitivity results are accurate, and no extra Monte Carlo simulations are needed, which is a computational advantage. In the case of sensitivity with respect 
FIGURE 7 Derivatives of EE over time for OT options.
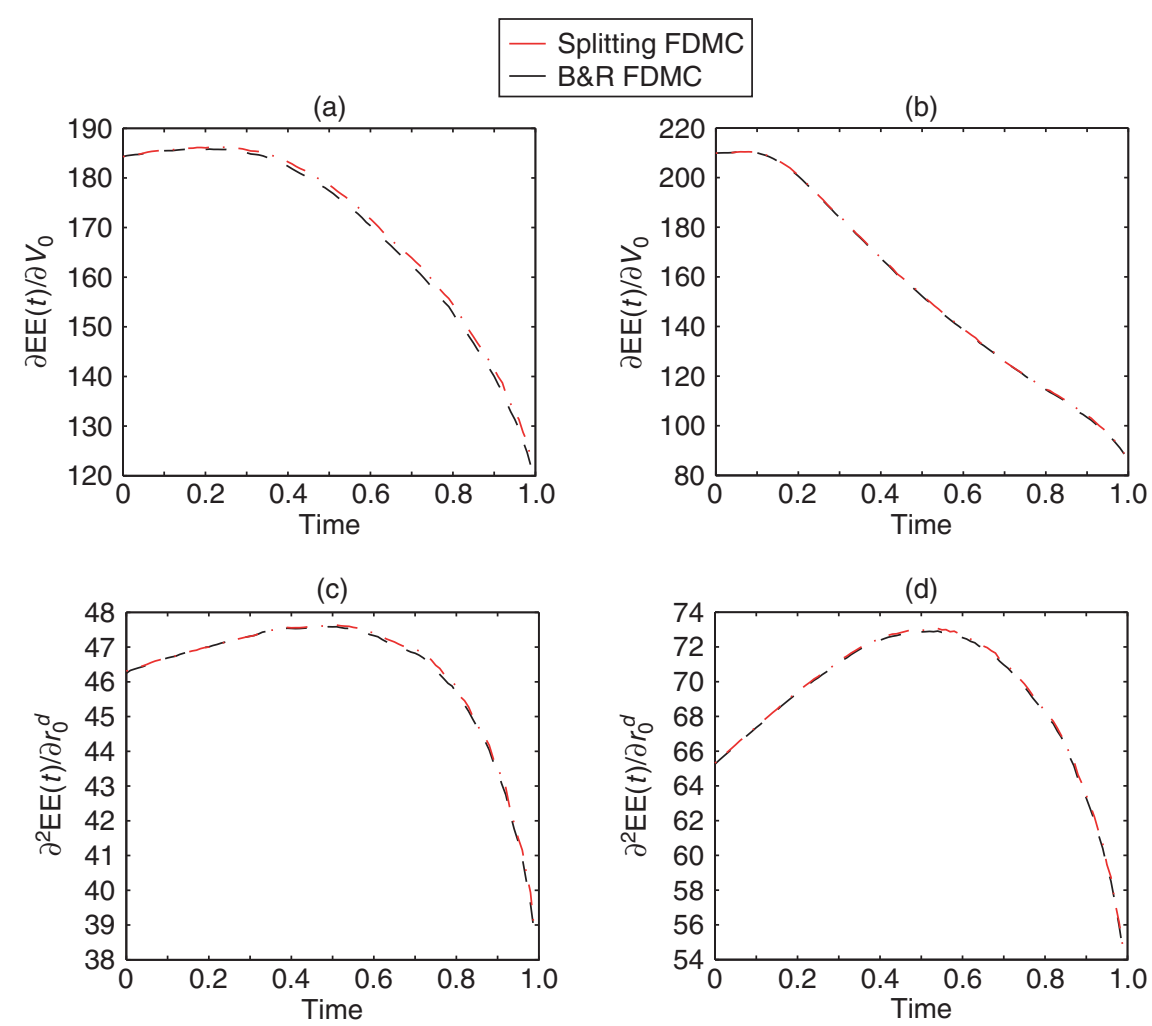

The derivatives are with respect to $S_{0}, V_{0}$ and $r_{0}^{\mathrm{d}}$. The sensitivities are computed by the splitting method and the bump-and-revalue method. We use 100 time points, and the number of finite difference grid points is set equal to 100 in the $s$-direction and 50 in the $v$ - and $r^{d}$ directions. (a) Vega profile for test A. (b) Vega profile for test B. (c) Rho profile for test $A$. (d) Rho profile for test $B$.

to $V_{0}$, we show that when an analytic expression of the future variance with respect to initial variance is available, the same technique can be applied.

Under the Heston dynamics, we analyzed the accuracy of the method by comparing it with a benchmark solution, and we assess the convergence of the solutions by (1) increasing the number of paths in the Monte Carlo simulation and (2) increasing the number of grid points used in the finite difference procedure. As expected, the standard error converges by $1 / \sqrt{N}$, where $N$ is the number of paths. By increasing the number of grid points, the relative error converges in first order.

Next, we showed that we can use the method to compute exposure profiles for a portfolio of options with different maturities. In this portfolio, the EEs of all options 
TABLE 7 Relative $L_{2}$ and $L_{\infty}$ differences between future exposure sensitivities, computed using a splitting scheme and a bump-and-revalue technique for one-year OT option, with barrier level $1.2 S_{0}$ and payout 100 .

\begin{tabular}{lccc}
\hline Difference & Quantity & Test A & Test B \\
\hline$\|\cdot\|_{\infty}$ & $\frac{\partial \mathrm{EE}}{\partial V_{0}}$ & $1.141 \times 10^{-2}$ & $4.325 \times 10^{-3}$ \\
& $\frac{\partial \mathrm{EE}}{\partial R_{0}^{\mathrm{d}}}$ & $6.872 \times 10^{-3}$ & $3.922 \times 10^{-3}$ \\
$\|\cdot\|_{2}$ & $\frac{\partial \mathrm{EE}}{\partial V_{0}}$ & $6.582 \times 10^{-3}$ & $2.478 \times 10^{-3}$ \\
& $\frac{\partial \mathrm{EE}}{\partial R_{0}^{\mathrm{d}}}$ & $1.946 \times 10^{-3}$ & $1.813 \times 10^{-3}$ \\
\hline
\end{tabular}

that are not path-dependent (European options) can be efficiently computed on a single grid. The resulting discontinuity in time is captured, and no significant error propagation is observed. The EEs for path-dependent options have to be computed individually and are added to the portfolio before computing the means. The sensitivities can again be computed with small extra computational time. Results compared with the Monte Carlo COS method are accurate for both linear and spline interpolation.

To further assess the impact of the interpolation, OT options that have a discontinuity over time are considered. In combination with the computationally challenging Heston Hull-White model, where less grid points can be used, the interpolation is essential. We found that even in this case a linear interpolation is sufficient, as differences are smaller than $1.2 \%$ for both exposures and sensitivities.

In forthcoming research, we assess in detail the effect of skew and stochastic interest rate on CVA and its sensitivities by using model parameters calibrated to real market data and a wide range of option contract parameters.

\section{DECLARATION OF INTEREST}

The authors report no conflict of interest. The authors alone are responsible for the writing of this work. This work was supported by the Dutch Technology Foundation STW under project 12214. Peter Sloot acknowledges support from the Russian Scientific foundation, Grant 14-21-00137.

\section{ACKNOWLEDGEMENTS}

We would like to thank Qian Feng for helpful advice regarding the Monte Carlo COS 
method. Further, we thank Norbert Hari, Shashi Jain and Sarunas Simaitis for useful discussions about the FDMC method.

\section{REFERENCES}

Albrecher, H., Mayer, P., Schoutens, W., and Tistaert, J. (2007). The little Heston trap. Wilmott Magazine, January, 83-92.

Andersen, L. (2008). Simple and efficient simulation of the Heston stochastic volatility model. The Journal of Computational Finance 11(3), 1-42 (http://doi.org/bm4f).

Basel Committee on Banking Supervision (2010). Basel III: a global regulatory framework for more resilient banks and banking systems. Technical Report, June.

Broadie, M., and Glasserman, P. (1996). Estimating security price derivatives using simulation. Management Science 42(2), 269-285 (http://doi.org/bmjd96).

Chan, J. H., and Joshi, M. (2010). First and second order Greeks in the Heston model. Working Paper 1718102, Social Science Research Network (http://doi.org/fzp474).

de Graaf, C. S. L., Feng, Q., Kandhai, B. D., and Oosterlee, C. W. (2014). Efficient computation of exposure profiles for counterparty credit risk. International Journal of Theoretical and Applied Finance 17(4), 1-24 (http://doi.org/bm4g).

Ekström, E., and Tysk, J. (2011). Boundary conditions for the single-factor term structure equation. Annals of Applied Probability 21(1), 332-350 (http://doi.org/cwhvxv).

Fang, F., and Oosterlee, C. W. (2011). A Fourier-based valuation method for Bermudan and barrier options under Heston's model. SIAM Journal on Financial Mathematics 2(1), 439-463 (http://doi.org/b2pshv).

Feng, Q., and Oosterlee, C. W. (2014). Monte Carlo calculation of exposure profiles and Greeks for Bermudan and barrier options under the Heston Hull-White model. Working Paper 2494233, Social Science Research Network (http://doi.org/bm4h).

Gregory, J. (2010). Counterparty Credit Risk. Wiley Online Library.

Guo, S., Grzelak, L. A., and Oosterlee, C. W. (2013). Analysis of an affine version of the Heston Hull-White option pricing partial differential equation. Applied Numerical Mathematics 72(October), 143-159 (http://doi.org/bm4j).

Haentjens, T., and In't Hout, K. J. (2012). ADI finite difference schemes for the Heston HullWhite PDE. The Journal of Computational Finance 16(1), 83-110 (http://doi.org/bm4k).

Heston, S. L. (1993). A closed-form solution for options with stochastic volatility with applications to bond and currency options. Review of Financial Studies 6(2), 327-343 (http://doi.org/fg525s).

Hull, J., and White, A. (1993). One-factor interest-rate models and the valuation of interestrate derivative securities. Journal of Financial and Quantitative Analysis 28(2), 235-254 (http://doi.org/bkbdm6).

Hull, J., and White, A. (2012). CVA and wrong-way risk. Financial Analysts Journal 68(5), 58-69 (http://doi.org/bm4m).

Hundsdorfer, W., and Verwer, J. G. (2003). Numerical Solution of Time-Dependent Advection-Diffusion-Reaction Equations, Volume 33. Springer Series in Computational Mathematics. Springer (http://doi.org/bm4n).

In't Hout, K. J., and Foulon, S. (2010). ADI finite difference schemes for option pricing. International Journal of Numerical Analysis and Modeling 7(2), 303-320. 
In't Hout, K. J., and Welfert, B. D. (2009). Unconditional stability of second-order ADI schemes applied to multi-dimensional diffusion equations with mixed derivative terms. Applied Numerical Mathematics 59(3-4), 677-692 (http://doi.org/dg3hrh).

$\mathrm{Ng}$, L., and Peterson, D. (2009). Potential future exposure calculations using the BGM model. Wilmott Journal 1(4), 213-225 (http://doi.org/c8ptbz).

Ng, L., Peterson, D., and Rodriguez, A. E. (2010). Potential future exposure calculations of multi-asset exotic products using the stochastic mesh method. The Journal of Computational Finance 14(2), 119-153 (http://doi.org/bm4p).

Schoutens, W., Simons, E., and Tistaert, J. (2004). A perfect calibration! Now what? Wilmott Magazine, March, 66-78.

Shen, Y., Weide, J. A. M., and Anderluh, J. H. M. (2013). A benchmark approach of counterparty credit exposure of Bermudan option under Lévy process: the Monte Carlo-COS method. Procedia Computer Science 18, 1163-1171 (http://doi.org/bm4q).

Tavella, D., and Randall, C. (2000). Pricing Financial Instruments: The Finite Difference Method. Wiley.

Whetten, M., Adelson, M., and Bemmelen, M. V. (2004). Credit default swap (CDS) primer. Nomura Fixed Income Research, April, 1-12. 